\title{
Tagungsbericht
}

\section{Rainer Kuhlen* \\ Ein nutzerfreundliche Urheberrecht für Bildung und Wissenschaft in Sicht}

DOI 10.1515/iwp-2016-0003

Im Mittelpunkt der Vorträge und Diskussionen zum Thema „Sind Bildung, Wissenschaft und Vergütung europäische oder nationale Aspekte des Urheberrechts?" stand beim Workshop des Aktionsbündnisses Urheberrecht für Bildung und Wissenschaft am 15. Oktober 2015 in den Räumen der Wikimedia-Stiftung in Berlin die Allgemeine Bildungsund Wissenschaftsklausel/-Schranke (ABWK/S). Einige Zeichen deuten darauf hin, dass in der EU und in Deutschland ein gerade für Bildung und Wissenschaft nutzerfreundlicheres Urheberrecht entstehen könnte. Das könnte durch eine ABWK/S erreicht werden, welche die bisherigen unzureichenden, nicht mehr zeitgemäßen Schrankenregelungen ersetzen sollte.

Die Vortragseinladung hatten sehr renommierte und auf die Themen des Workshops spezialisierte Personen angenommen, deren Vorträge mit großem Interesse aufgenommen und ausführlich diskutiert wurden.

Julia Reda, international bekannt geworden durch ihren schließlich vom Parlament angenommen Reda-Report, vermittelte einen Einblick in den schwierigen zeitaufwändigen Weg durch die EU-Institutionen. So schnell, wie es einmal Jean-Claude Juncker, Präsident der Europäischen Kommission, angekündigt hatte, wird es kaum gehen. Reformentwürfe mit Blick auf Bildung und Wissenschaft könnten vielleicht erst in zwei Jahren entscheidungsreif sein. Frau Reda sah jedoch eine Chance, dass Deutschland durch rasche Verabschiedung einer im Koalitionsvertrag der jetzigen Regierung vorgesehenen Allgemeinen Bildungs- und Wissenschaftsschranke (ABWS) ein Vorbild für die EU-Entwicklung und andere Länder geben könnte.

Prof. Senftleben von der Universität Amsterdam erläuterte den von Gesetzgebung und Rechtsprechung als „Bremse“ für Schrankenregelungen verwendeten Dreistufentest. Nach diesem Test sollten Schranken nur in „bestimmten Sonderfällen“ (1) erlaubt sein und dürfen weder die „normale Verwertung“ (2) noch die „berechtigten Interessen der Verwerter und Urheber“ (3) ungebührlich ver-

*Kontaktperson: Prof.-em. Dr. Rainer Kuhlen, Bogotastraße 4, 14163 Berlin, E-Mail: rainer.kuhlen@uni-konstanz.de letzen. Prof. Senftleben stellte klar, dass dieser Test im internationalen Rahmen ursprünglich eher als Ermöglichungs- und nicht als Verhinderungsinstrument gedacht war. Diesen Freiraum gelte es wieder zurückzugewinnen. Nicht zuletzt durch die Entwicklungen im Internet und besonders im Publikationsgeschehen (Open Access!) müsste es zu einer sehr viel offeneren und vor allem Bildung und Wissenschaft begünstigenderen Interpretation dieses Tests kommen. Prof. Senftleben stellte dann auch einen Vorschlag für eine ABWS vor, durch den Nutzungen für Ausbildung und Forschung gerade durch eine liberalere Interpretation des Dreistufentests legitimiert werden.

Prof. Kuhlen verzichtete auf eine Vorstellung des Vorschlags des Aktionsbündnisses für eine ABWK (dazu ist ein Faltblatt abrufbar unter http://www.urheberrechtsbu endnis.de/docs/abwk-flyer-2015-a4.pdf) und konzentrierte sich auf den ersten Satz (Abs. 1, Satz 1), der als Generalklausel zu verstehen ist: Die Nutzung publizierter Werke ist in Bildung und Wissenschaft für die (nicht-kommerziellen) Zwecke von Lehre, Studium/Lernen und Forschung genehmigungsfrei erlaubt. Eine solche ABWK würde die in Bildung und Wissenschaft Tätigen von der mühsamen und letztlich oft hoffnungslosen Auseinandersetzung mit den im Urheberrechtsgesetz verstreuten verschiedenen Schrankenregelungen befreien. Kuhlen betonte, dass im Sinne des selbstbestimmten Lernens vor allem in E-Learning-Umgebungen auch die Nutzung durch Lernende über die Erlaubnis der Privatkopie in $\S 53$ UrhG hinaus privilegiert werden sollte.

Im Rahmen einer ABWK/S sollten auch die verschiedenen schwer verständlichen Einschränkungen der Schranken beseitigt werden. Diese sind kaum praxistauglich und können zurzeit - immer wieder unterschiedlich - bezüglich des Umfangs und des Ortes der Nutzung und der zulässigen Weiterverarbeitung (Digitalisieren, Speichern und Ausdrucken) interpretiert werden. Eine ABWS/K, wenn sie denn wirklich Nutzen stiften sollte, dürfe sich nicht mehr strikt an den jetzigen Vorgaben der Rechtsetzung und -sprechung orientieren, sondern versuchen, den jetzt gültigen Rahmen kreativ zu verstehen und ggfls. auch zu erweitern. Die oberen Gerichte, wie BGH und EuGH, haben in der Vergangenheit diese kreative Auslegung verschiedentlich 
praktiziert. Begrüßt wurde von allen Beteiligten, dass durch die jüngsten Entscheidungen das Kriterium des „geboten" nicht so weit ausgelegt werden darf, dass ein bloßes Verlagsangebot die rechtlichen Regelungen einer Schrankenbestimmung aushebeln kann.

Bezüglich der Vergütung will das Aktionsbündnis auch das bisherige Verfahren auf den Prüfstand stellen. $\mathrm{Zu}$ hinterfragen ist, ob ein Vergütungsanspruch an Werken im Gesetz festgeschrieben werden muss, wenn diese durch öffentliche Finanzierung und durch Personen entstanden sind, die von der Öffentlichkeit finanziert werden. Faktisch kann dieser Anspruch zumeist sowieso nicht von den Urhebern gegenüber den Verlagen durchgesetzt werden. Oft wird eine Meldung an eine Verwertungsgesellschaft unterlassen, weil der Aufwand dafür als zu hoch empfunden wird. Falls es doch zu Vergütungen kommen muss, hält das Aktionsbündnis nur Pauschalabrechnungsverfahren und keinesfalls die Individualabrechnung für sinnvoll und akzeptabel. Die bislang in der Rechtsprechung allein für rechtens erklärte Individualabrechnung scheitert an der praktischen Umsetzbarkeit.

Prof. Kallenrode von der Universität Osnabrück bestätigte durch eindrucksvolle Daten diese Einschätzung. Sie war als Vizepräsidentin der Universität Osnabrück für das Management einer Machbarkeitsstudie zuständig, durch die Kennzahlen für Kosten, Aufwand und Workflows bei der Einzelerfassung und Abrechnung von Lehrmaterialien (entsprechend den Vorgaben von $\S 52 \mathrm{a}$ UrhG) ermittelt wurden. Das Fazit ist eindeutig: Sowohl für die Lehrenden als auch für die Studierenden ist der Aufwand für die Einzelerfassung weder zumutbar noch zu rechtfertigen. Sie wirkt abschreckend und führt zu einem Rückgang bei der Bereitstellung von Materialien in der Lehre und damit auch zu Einbußen in deren Qualität. Administrativ entsteht ein unverhältnismäßig hoher finanzieller Aufwand für die Durchführung der Melde- und Abrechnungsvorgaben - unverhältnismäßig auch angesichts des geringen Betrags, der auf Grund von meldepflichtigen Nutzungen an die VG Wort gezahlt werden musste. Alle Beteiligten forderten eine Rückkehr zur Pauschalvergütung über Gesamtverträge.

Das war auch der Tenor in der Gesamtdiskussion, einschließlich aller Referenten. Die Vorgabe des BGH für Einzelvergütung sei nicht haltbar und auch unnötig. Vergütung im Rahmen einer ABWS darf nicht auf der Grundlage einer Einzelabrechnung erfolgen.

Ein Lizenzierungs- und Abrechnungsverfahren initiiert aus der Verlagswirtschaft stellte Dr. Salzmann von Booktex vor. Er demonstrierte, wie mit dem Anfang 2015 gestarteten Angebot des Verlagskonsortiums Booktex GmbH elektronische Verlagsmaterialien in Lernplattformen individuell lizenziert werden können. Das Angebot bezieht sich derzeit auf ca. 40.000 deutschsprachige Werke. Inwieweit es sich hierbei jeweils um angemessene Verlagsangebote handelt, bleibt der Entscheidung eines jeden Nutzers bzw. jeder Bibliothek vorbehalten. Nach der aktuellen Rechtsprechung (zu § 52b UrhG) müssen Nutzer oder Vermittlungseinrichtungen wie Bibliotheken solche Lizenzangebote nicht annehmen. Man wird sehen, ob sich der Markt durch Lizenzierungsangebote durchsetzen kann oder ob anderen Nutzungsformen durch rechtlich verbindliche Regelungen wie durch eine ABWS/Kattraktiver und realitätsnäher sind.

Prof. Peukert von der Goethe-Universität Frankfurt am Main setzte unter dem programmatischen Titel „Informationsfreiheit für Bildung und Wissenschaft durch vergütungsfreie Schranken des Urheberrechts“ den Schlusspunkt. Er nahm die in der ABWK des Aktionsbündnisses vorgesehenen Regelungen Punkt für Punkt genau, kritisch und äußerst kreativ-konstruktiv unter die Lupe. Folgenreich könnte es u.a. sein, dass selbst in der InfoSocRichtlinie von 2001 nicht in allen Fällen eine Vergütungspflicht vorgesehen ist (z. B. für die Nutzung an den „Leseplätzen“ in den Bibliotheken). Für Prof. Peukert könnte sich eine neue Diskussionsgrundlage dadurch ergeben, dass das Argument der öffentlichen Finanzierung von Werken und Forschern bislang nicht angemessen berücksichtigt wurde. Ohnehin deutet auch die Open-AccessEntwicklung (einschließlich deren möglichen Mandatierung) darauf hin, dass Vergütung im Bereich Bildung und Wissenschaft bis auf wenige Ausnahme nicht die entscheidende Rolle spielt und deshalb nicht weiterhin im Recht verbindlich geregelt werden muss.

Wesentliches Ergebnis der Tagung und der Diskussion in der Vollversammlung ist, dass in der Auseinandersetzung um eine ABWS/K in Deutschland weniger auf einen konkreten Normentwurf (wie den des Aktionsbündnisses) beharrt werden sollte, sondern dass vielmehr ein Katalog von Zielvorstellungen für eine ABWS/K erarbeitet und in der Öffentlichkeit und Politik kommuniziert werden sollte. Das könnten dann auch Prüfkriterien für den Referentenentwurf des BMJV sein.

Deskriptoren: Tagung, Urheberrecht, Deutschland, Europa, Wissenschaft und Technik, Bildungswesen

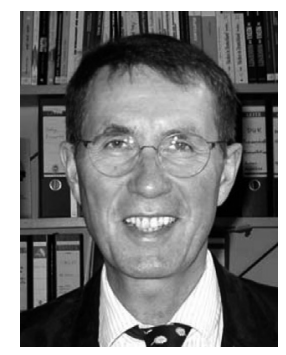

Prof.-em. Dr. Rainer Kuhlen

Bogotastraße 4

14163 Berlin

rainer.kuhlen@uni-konstanz.de 